OPEN ACCESS

Edited by:

Hyesung Park,

Ulsan National Institute of Science and

Technology, South Korea

Reviewed by: Jacopo Parravicini,

University of Milano-Bicocca, Italy

Han Zhang,

Shenzhen University, China

*Correspondence:

Guobao Jiang

andguobao@163.com

Specialty section

This article was submitted to

Optics and Photonics,

a section of the journal

Frontiers in Physics

Received: 11 November 2019

Accepted: 23 March 2020

Published: 21 April 2020

Citation:

Wu M and Jiang G (2020) Observation the Multi-Soliton Patterns From the

Er-Doped Mode-Locked Fiber Laser Modulated by PtSe

Front. Phys. 8:107.

doi: 10.3389/fphy.2020.00107

\section{Observation the Multi-Soliton Patterns From the Er-Doped Mode-Locked Fiber Laser Modulated by $\mathrm{PtSe}_{2}$}

\author{
Man $W u^{1}$ and Guobao Jiang ${ }^{2 *}$ \\ ${ }^{1}$ School of Physics and Electronic Science, Hunan University of Science and Technology, Xiangtan, China, ${ }^{2}$ School of \\ Electronic Information and Electrical Engineering, Changsha University, Changsha, China
}

The passively mode-locked fiber laser modulated by low-dimensional materials can provide an ideal platform to investigate the soliton dynamics. Here, we experimentally explored the soliton formation and evolution from an erbium-doped fiber laser mode-locked by mechanically exfoliated $\mathrm{PtSe}_{2}$ saturable absorber via evanescent field interaction. With the increasing pump power, different soliton patterns, such as single soliton, two solitons, and bound soliton, can be observed experimentally. The experimental results can identify the non-linear optical response of the $\mathrm{PtSe}_{2}$ and may make avenue toward unveiling the nature of the soliton dynamics in the fiber laser system.

Keywords: non-linear optics, soliton dynamics, mode-locking, fiber laser, saturable absorber

\section{INTRODUCTION}

Transient phenomena and ultrafast dynamics are very important for understanding and unveiling the nature of the non-linear systems. Soliton, a localized wave structure, can be formed and observed in different non-linear processes, such as hydrodynamics, biology dynamics, plasma physics, and optics [1-3]. Among the separate optical systems, the passively mode-locked fiber lasers can deliver robust ultrashort pulses with a compact and stable configuration. In addition, the passively mode-locked fiber lasers can provide an ideal platform to investigate soliton evolution behaviors [1-5]. Ordinarily, the soliton pulse will favor multiple pulsing with increasing pump power for the peak power-limiting effect in a passively mode-locked fiber laser operating in the anomalous dispersion regime [1,2]. With different cavity arrangements and pump power, different pulse patterns can be observed, such as single soliton, multi-solitons [3], and bound soliton $[4,5]$, etc.

The ultrashort pulse can be generated from the passively mode-locked fiber laser with the key component, i.e., saturable absorber (SA). The SA can be divided into the artificial SA [such as non-linear polarization rotation (NPR) technique [6], the figure-eight-cavity [7] method], the real SA [such as semiconductor saturable absorber mirrors (SESAMs) [8], carbon nanotubes (CNTs) [9], and low-dimensional materials [10-16]], and the hybrid type. However, the modulation depth and operating wavelength of SESAMs are small and narrow. The non-linear absorption of CNTs 
depends on the size and chirality, which would affect the laser performance. Graphene has a small linear absorption coefficient and limited modulation depth, which will influence the stability and pulse narrowing of the laser. With the functional material evolvement, novel low-dimensional materials have shown excellent non-linear optical performance, such as the hybrid perovskite [17], metal-organic framework [18], and versatile transition metal dichalcogenides (TMDs) [19-22].

TMDs have caught researchers' attention due to their unique properties in both physics and chemistry [20-22]. In respect of non-linear optics, the TMDs have been applied as SA in pulsed laser generation. However, the non-linear threshold in the optical communication wavelength is too large for the large bandgap of the usual TMDs [23]. In recent years, two newly emerged TMDs, $\mathrm{PtS}_{2}$ and $\mathrm{PtSe}_{2}$, have been studied for their layer-dependent bandgap largely tuned from 1.2 to $0.2 \mathrm{eV}$ [24, 25], which can cover nearly all of the optical communication band. The outstanding high-performance devices based on $\mathrm{PtSe}_{2}$ have been investigated, such as the pressure and gas sensors [26, 27], broadband mid-infrared photodetector [28], and wide spectral photoresponse photodiode [29]. In addition, the $\mathrm{PtSe}_{2}$ has advantages of high carrier mobility [30-32], ambient stable and sizable band gap, all of which filled the gaps of the materials mentioned above. Moreover, $\mathrm{PtSe}_{2}$ has excellent non-linear optical response, which can provide a candidate to explore the soliton evolution in the passively mode-locked fiber laser.

Here, $\mathrm{PtSe}_{2}$ has been prepared via a mechanical exfoliation method, and then transferred onto the side-polished fiber to form a SA. With the SA, we have observed different kinds of solitons, such as single soliton, two solitons, bound soliton, and noise-like pulse, from the fiber laser by tuning the pump power or the polarization controllers. By optimizing the cavity, the fiber laser can deliver the shortest pulse duration down to 699 fs with a signal-to-noise ratio of $57 \mathrm{~dB}$ and repetition rate of $11.26 \mathrm{MHz}$.

\section{EXPERIMENTS AND RESULTS}

The bulked $\mathrm{PtSe}_{2}$ crystal is bought from HQ Graphene Company. The mechanical exfoliation method was used to exfoliate $\mathrm{PtSe}_{2}$ [33], which was then transferred onto the D-shaped fiber to form a SA. It is an easy, effective and low-cost exfoliation method to prepare the $\mathrm{PtSe}_{2}$ based SA. To characterize the exfoliated $\mathrm{PtSe}_{2}$, we have carried out the scanning electronic microscope (SEM) and Raman spectra measurements. The Raman spectrum of $\mathrm{PtSe}_{2}$ is shown in Figure 1A, which has two Raman peaks at 175 and $205 \mathrm{~cm}^{-1}$ assigned to $\mathrm{E}_{\mathrm{g}}$ and $\mathrm{A}_{1 \mathrm{~g}}$ modes shown in Figure 1A, matching well with the results of previous work [34]. The inset of the Figure 1A is the SEM of $\mathrm{PtSe}_{2}$, from which it can be inferred that the mechanically exfoliated $\mathrm{PtSe}_{2}$ is relatively smooth. The twin-detector method has been performed to test the non-linear optical absorption of $\mathrm{PtSe}_{2}$. The adopted laser for measurement has the center wavelength $1,560 \mathrm{~nm}$, pulse width $130 \mathrm{ps}$, repetition rate 20.8 $\mathrm{MHz}$ [34]. The non-linear absorption curve of $\mathrm{PtSe}_{2}$ has been shown in Figure 1B. From the plot, we can see that the SA has a modulation depth $6.8 \%$ and a saturation intensity 9.9 $\mathrm{MW} / \mathrm{cm}^{2}$, respectively.

To verify the non-linear absorption of $\mathrm{PtSe}_{2}$, a fiber laser was constructed, as shown in Figure 2. The passively modelocked fiber laser operating in a fiber ring cavity with a length of $17.5 \mathrm{~m}$ includes a $16.6 \mathrm{~m}$ standard passive single mode fiber (SMF-28) and a $0.9 \mathrm{~m}$ active erbium-doped fiber (EDF, LIEKKI Er 80-8/125). A laser diode with a central wavelength of $975 \mathrm{~nm}$ is adopted as the pump laser, which is introduced into the fiber ring cavity via a 980/1,550 wavelength-division multiplexer (WDM). The laser can be delivered out from the cavity via a $15 \%$ output coupler. A polarization independent isolator (PI-ISO) is fused between the D-shaped fiber and polarization controllers (PCs) to enforce the unidirectional operation of the intracavity laser. During the experiments, the PCs can be adjusted to tune the polarization state of the light circulating in the cavity [34].

The $\mathrm{PtSe} \mathrm{C}_{2}$ based SA was brought into the fiber ring cavity to act as a non-linear optical modulator. Firstly, self-started Q-switched mode-locking operation was achieved by increasing the pump power up to $160 \mathrm{~mW}$. Figure 3A has shown the corresponding optical spectrum of Q-switched mode-locking, from which we can infer that the output wavelength is centered at $1,567 \mathrm{~nm}$. The typical pulse trace of Q-switched mode-locking output is shown in Figure 3B, from which we can see that the mode-locked pulses enveloped form a Q-switched pulse shape.

When the pump power is increased up to $180 \mathrm{~mW}$, the output pulse changes to the mode-locked regime, as shown in Figure 4. Figure 4A shows the corresponding optical spectrum with a central wavelength of $1,566 \mathrm{~nm}$, and $3 \mathrm{~dB}$ spectral bandwidth about $3.1 \mathrm{~nm}$. By enlarging the span of the oscilloscope trace, we can see the mode-locked pulse over $2 \mu$ s time scale, as shown in Figure 4B. It can be seen from the figure that the soliton begins to split due to a soliton peak clamping effect and pulse shaping of the dispersive waves with increasing intra-cavity energy [1]. We can also obtain the full width at half maximum (FWHM) of the pulse duration 1.139 ps in Figure 4C, which indicates a true pulse width of about $699 \mathrm{fs}$, fitted by the hyperbolic secant function $\left(\operatorname{sech}^{2}\right)$. Figure 4D shows the electrical spectrum of the modelocked pulse with signal-to-noise ratio (SNR) up to $57 \mathrm{~dB}$, which indicates a stable mode-locking operation. In addition, we have not observed extra frequency components within a wide radio frequency spectrum up to $500 \mathrm{MHz}$ in the inset of Figure 4D.

By further increasing the pump power to $210 \mathrm{~mW}$, the single soliton will split into two solitons. As can be seen in Figure 5A, the mode-locked pulse breaks up easily due to the soliton quantization effect arising from the two-photon absorption. The repetition rate doubled in Figure 5B implies that the soliton pulse breaks into two pulses.

Under the same pump conditions, the laser pulse can be switched from the traditional mode-locked soliton to bound soliton by slightly adjusting the PC. The optical spectrum with sidebands has been shown in Figure 6A, which is the typical symbol of the two-pulse bound soliton. The bound soliton locates at about $1,565 \mathrm{~nm}$ wavelength with spectral modulation period of $2 \mathrm{~nm}$ [1,35-38]. Three peaks can be 

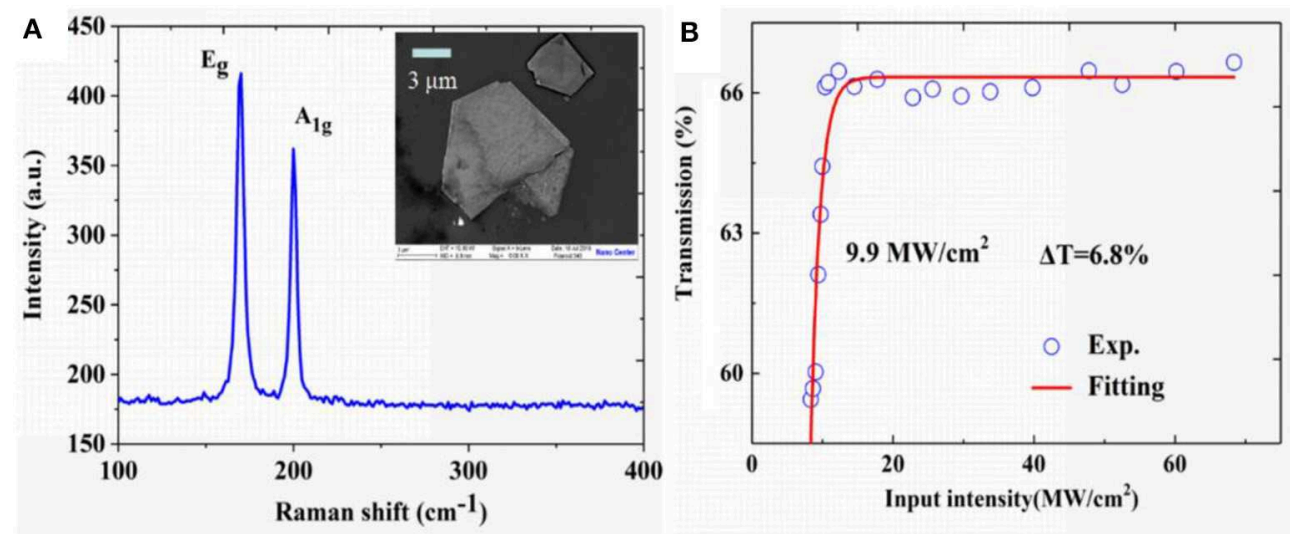

FIGURE 1 | (A) Raman spectrum of $\mathrm{PtSe}_{2}$ (The inset is the SEM of PtSe ${ }_{2}$ ). (B) The non-linear optical absorption curve of PtSe ${ }_{2}$.

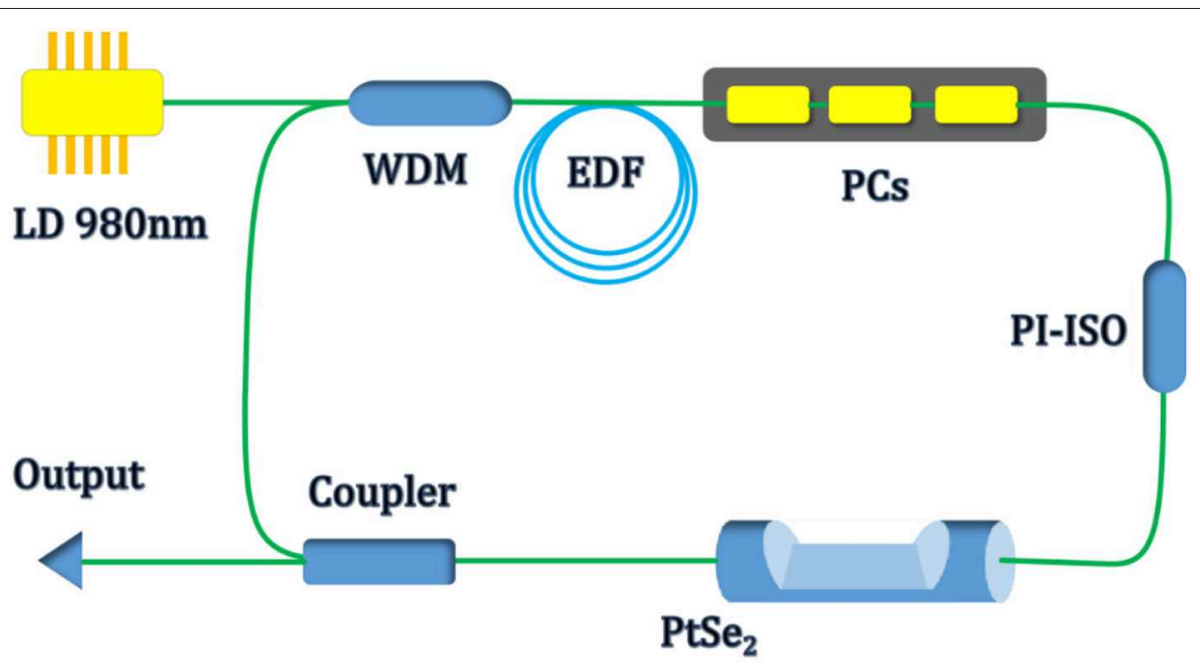

FIGURE 2 | Experimental setup of the mode-locked Er-doped fiber laser.
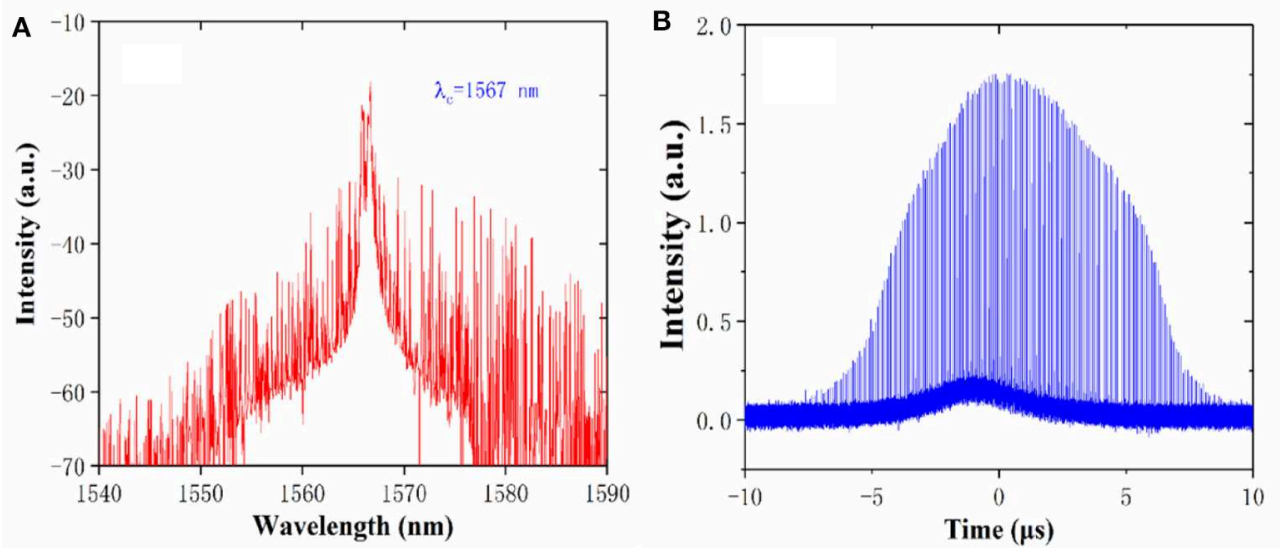

FIGURE 3 | (A) Output spectrum. (B) Q-switched mode-locked pulse trace. 

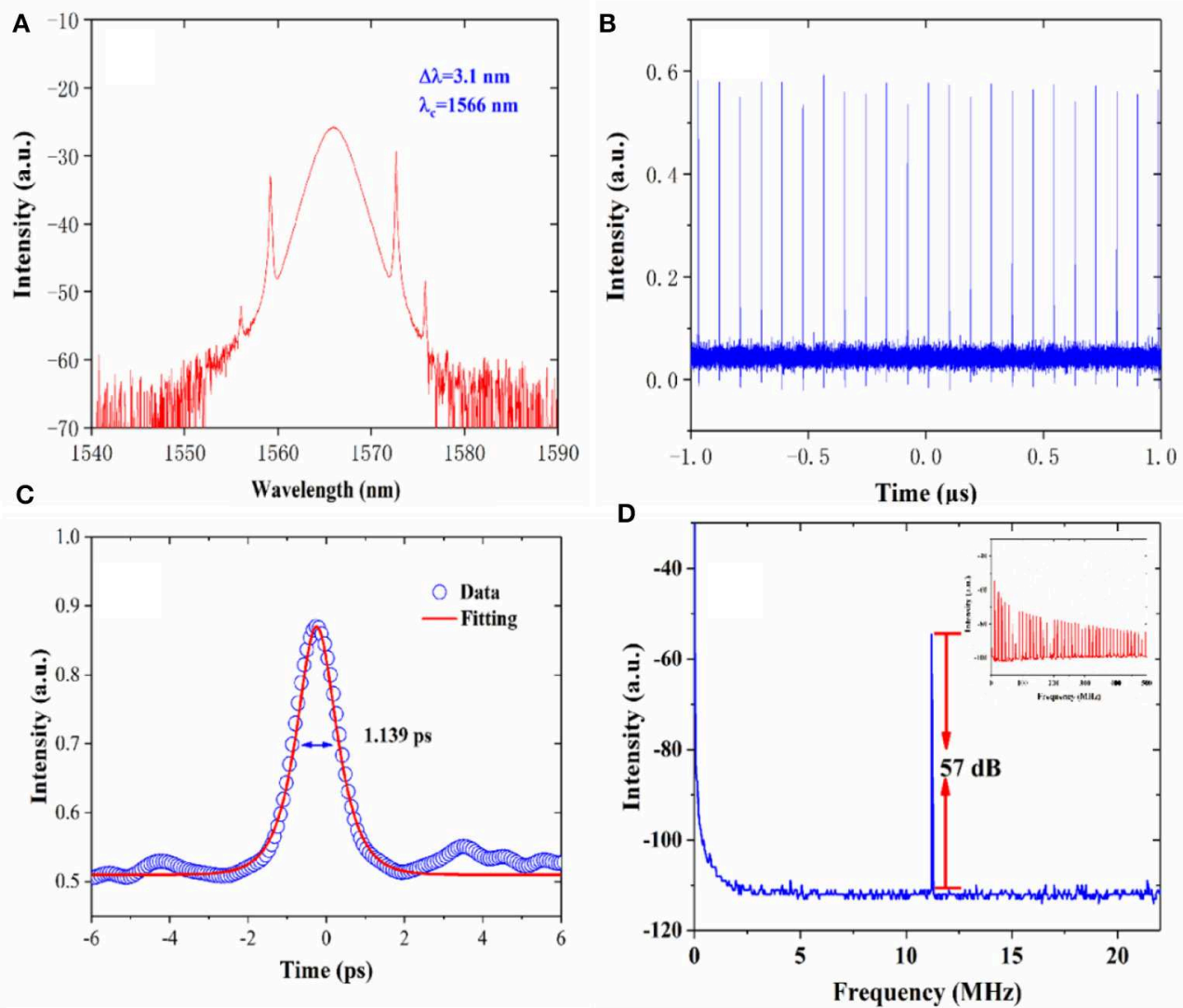

FIGURE 4 | (A) Output spectrum of single soliton. (B) Pulse train of single soliton. (C) Autocorrelation trace. (D) Radio frequency spectrum (Inset: wide-band RF spectrum)
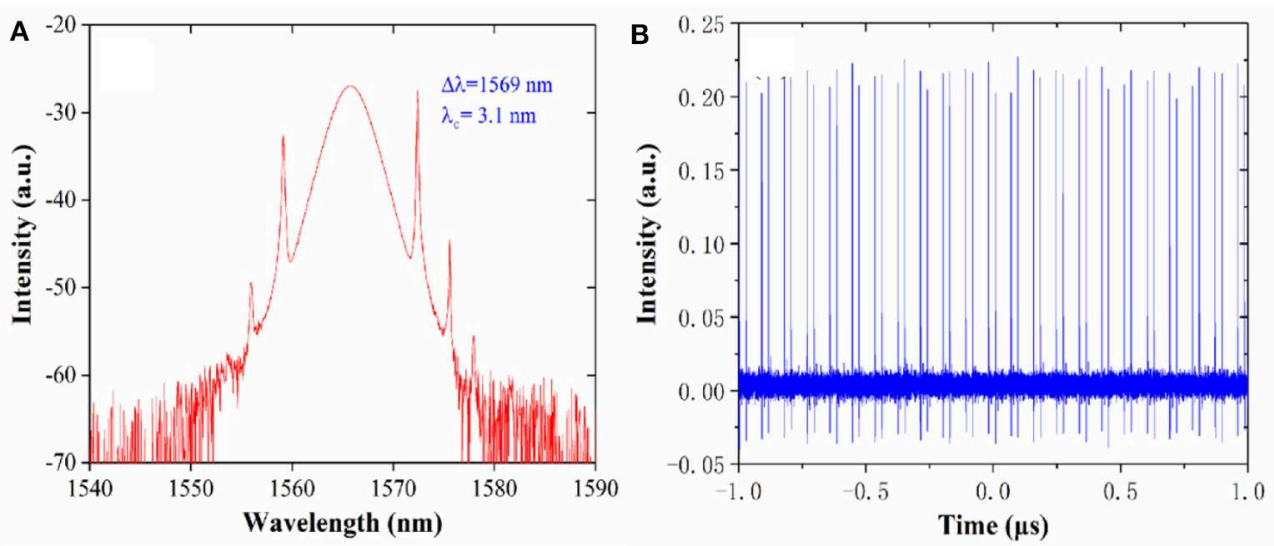

FIGURE 5 | (A) Output spectrum. (B) Pulse train.

seen in the autocorrelation trace in Figure 6B, which means that the bound soliton is a two phase-locking pulse. The oscilloscope trace in Figure 6C also confirmed the bound soliton operation [38].

\section{DISCUSSIONS}

The pulsed fiber laser can be easily achieved, which indicates that the $\mathrm{PtSe}_{2}$ based $\mathrm{SA}$ is a promising one. Due to the 

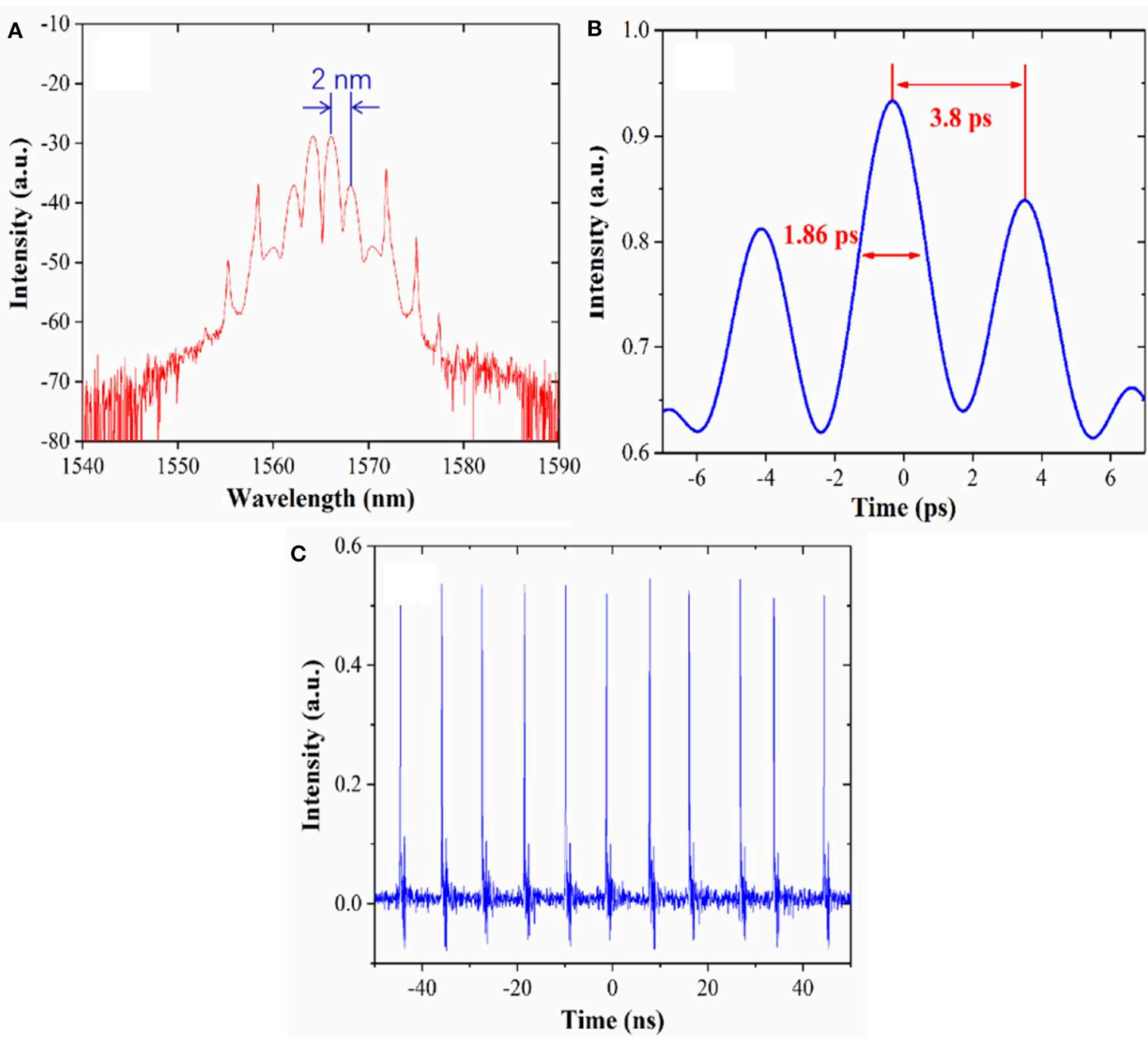

FIGURE 6 | (A) Output spectrum; (B) autocorrelation trace; (C) pulse train.

non-uniform distribution of $\mathrm{PtSe}_{2}$, which will weaken the interaction between materials and guided light, the pulsed output needs a relatively large pump power. However, once the pulsed output is obtained, the output can maintain long-term stability. From the experimental results, the $\mathrm{PtSe}_{2}$ has been proved to be a good candidate for ultrashort pulse modulation.

Based on the excellent non-linear optical response of $\mathrm{PtSe}_{2}$, the ultrashort pulses have been generated with different mechanisms [1, 35-37]. While the pump power is under the pulse shaping threshold, a Q-switched mode-locking pulse can be observed. In the mode-locking regime, a single soliton, two solitons, and a bound soliton have been obtained. Single soliton operation is the prevailing mode-locking operation in an anomalous dispersion laser cavity. Owing to the soliton quantization effect and peak limiting originating from the two-photon absorption effect [35], the soliton breaks up easily. When the pump power is set to $180 \mathrm{~mW}$, a two solitons mode-locking operation can be achieved. Two solitons transform separately in the laser cavity, unless the random relative-phase variations between the solitons are not suppressed [36]. The $1,565 \mathrm{~nm}$ wavelength bound soliton has been observed with a modulation period of $2 \mathrm{~nm}(\Delta \nu=244 \mathrm{GHz})$, as shown in Figure 6A, which indicates that the pulse-to-pulse interval is 4.1 ps. The autocorrelation trace in Figure 6B shows three peaks, implying that the bound soliton is a two phase-locking pulse [38].

\section{CONCLUSION}

We have prepared the $\mathrm{PtSe}_{2} \mathrm{SA}$ via the evanescent field coupling method by transferring the mechanically exfoliated $\mathrm{PtSe}_{2}$ onto the side-polished fiber. By implementing the $\mathrm{PtSe}_{2}$ based SA in a fiber ring laser, different solitons have been observed, and the mode-locked pulse has evolved from a single soliton to two solitons and a bound soliton by increasing the pump power. This work suggests that $\mathrm{PtSe} \mathrm{e}_{2}$ could be a promising $\mathrm{SA}$, and the layered material may provide an ideal platform to investigate the soliton formation and dynamics in fiber lasers. 


\section{DATA AVAILABILITY STATEMENT}

The raw data supporting the conclusions of this article will be made available by the authors, without undue reservation, to any qualified researcher.

\section{REFERENCES}

1. Tang DY, Zhao LM, Zhao B, Liu AQ. Mechanism of multisoliton formation and soliton energy quantization in passively mode-locked fiber lasers. Phys Rev A. (2005) 72:043816. doi: 10.1103/PhysRevA.72.043816

2. Grelu P, Belhache F, Gutty F, Soto-Crespo JM. Phase-locked soliton pairs in a stretched-pulse fiber laser. Opt Lett. (2002) 27:966-8. doi: 10.1364/OL.27.000966

3. Akhmediev NN, Ankiewicz A, Soto-Crespo JM. Multisoliton solutions of the complex Ginzburg-Landau equation. Phys Rev Lett. (1997) 79:4047. doi: 10.1103/PhysRevLett.79.4047

4. Wang Z, Zhan L, Majeed A, Zou Z. Harmonic mode locking of bound solitons. Opt Lett. (2015) 40:1065-8. doi: 10.1364/OL.40.001065

5. Malomed BA. Bound Solitons in the Nonlinear Schrödinger Ginzburg-Landau Equation. Large Scale Structures in Nonlinear Physics. Berlin; Heidelberg: Springer (1991) p. 288-94. doi: 10.1007/3-540-54899-8_48

6. Luo ZC, Luo AP, Xu WC, Song CX, Gao YX, Chen W. Sideband controllable soliton all-fiber ring laser passively mode-locked by nonlinear polarization rotation. Laser Phys Lett. (2009) 6:582-5. doi: 10.1002/lapl.200910032

7. Yun L, Liu X, Mao D. Observation of dual-wavelength dissipative solitons in a figure-eight erbium-doped fiber laser. Opt Express. (2012) 20:209927. doi: 10.1364/OE.20.020992

8. Zhao L, Tang D, Wu X, Zhang H, Tam HY. Coexistence of polarization-locked and polarization-rotating vector solitons in a fiber laser with SESAM. Opt Lett. (2009) 34:3059-61. doi: 10.1364/OL.34.003059

9. Kieu K, Wise FW. Soliton thulium-doped fiber laser with carbon nanotube saturable absorber. IEEE Photon. Technol Lett. (2008) 21:12830. doi: 10.1109/LPT.2008.2008727

10. Zhang H, Tang D, Knize R, Zhao L, Bao Q, Loh KP. Graphene mode locked, wavelength-tunable, dissipative soliton fiber laser. Appl Phys Lett. (2010) 96:111112. doi: 10.1063/1.3367743

11. Song Y, Chen S, Zhang Q, Li L, Zhao L, Zhang H, et al. Vector soliton fiber laser passively mode locked by few layer black phosphorus-based optical saturable absorber. Opt Express. (2016) 24:25933-42. doi: 10.1364/OE.24.025933

12. Chen Y, Jiang G, Chen S, Guo Z, Yu X, Zhao C, et al. Mechanically exfoliated black phosphorus as a new saturable absorber for both Qswitching and mode-locking laser operation. Opt Express. (2015) 23:1282333. doi: 10.1364/OE.23.012823

13. Wang ZT, Chen Y, Zhao CJ, Zhang H, Wen SC. Switchable dualwavelength synchronously Q-switched erbium-doped fiber laser based on graphene saturable absorber. IEEE Photon J. (2012) 4:869-76. doi: 10.1109/JPHOT.2012.2199102

14. Ma J, Lu SB, Guo ZN, Xu XD, Zhang H, Tang DY, et al. Few-layer black phosphorus based saturable absorber mirror for pulsed solid-state lasers. Opt Expess. (2015) 23:22643-8. doi: 10.1364/OE.23.022643

15. Zhao LM, Tang DY, Wu X, Zhang H. Dissipative soliton generation in Yb-fiber laser with an invisible intracavity bandpass filter. Opt Lett. (2010) 35:2756-8. doi: 10.1364/OL.35.002756

16. Lu L, Liang ZM, Wu LM, Chen YX, Song YF, Dhanabalan SC, et al. Fewlayer bismuthene: sonochemical exfoliation, nonlinear optics and applications for ultrafast photonics with enhanced stability. Laser Photon Rev. (2017) 12:1700221. doi: 10.1002/lpor.201700221

17. Zhang YP, Lim CK, Dai ZG, Yu GN, Haus JW, Zhang H, et al. Photonics and optoelectronics using nano-structured hybrid perovskite media and their optical cavities. Phys Rep. (2019) 795:1-52. doi: 10.1016/j.physrep.2019.01.005

18. Jiang XT, Zhang LJ, Liu SX, Zhang YY, He ZL, Li WJ, et al. Ultrathin metal-organic framework: an emerging broadband nonlinear optical material for ultrafast photonics. Adv Opt Mater. (2018) 6:1800561. doi: 10.1002/adom.201800561

\section{AUTHOR CONTRIBUTIONS}

GJ designed experiments. MW carried out experiments. GJ analyzed experimental results. MW and GJ wrote the manuscript.

19. Ge YQ, Zhu ZF, Xu YH, Chen YX, Chen S, Liang ZM, et al. Ultrafast photonics: broadband nonlinear photoresponse of $2 \mathrm{D} \mathrm{TiS}_{2}$ for ultrashort pulse generation and all-optical thresholding devices. Adv Opt Mater. (2018) 6:1870014. doi: 10.1002/adom.201870014

20. Wang QH, Kalantar-Zadeh K, Kis A, Coleman JN, Strano MS. Electronics and optoelectronics of two-dimensional transition metal dichalcogenides. Nat Nanotechnol. (2012) 7:699. doi: 10.1038/nnano.2012.193

21. Xia $F$, Wang $H$, Xiao D, Dubey $M$, Ramasubramaniam A. Two-dimensional material nanophotonics. Nat Photon. (2014) 8:899. doi: 10.1038/nphoton.2014.271

22. Chhowalla M, Shin HS, Eda G, Li LJ, Loh KP, Zhang H. The chemistry of twodimensional layered transition metal dichalcogenide nanosheets. Nat Chem. (2013) 5:263. doi: 10.1038/nchem.1589

23. Zhao W, Ribeiro RM, Toh M, Carvalho A, Kloc C, Castro Neto AH, et al. Origin of indirect optical transitions in few-layer $\mathrm{MoS}_{2}, \mathrm{WS}_{2}$, and $\mathrm{WSe}_{2}$. Nano Lett. (2013) 13:5627-34. doi: 10.1021/nl403270k

24. Wang Z, Li Q, Besenbacher F, Dong M. Facile synthesis of single crystal $\mathrm{PtSe}_{2}$ nanosheets for nanoscale electronics. Adv Mater. (2016) 28:102249. doi: 10.1002/adma.201602889

25. Wang Y, Li L, Yao W, Song S, Sun JT, Pan J, et al. Monolayer $\mathrm{PtSe}_{2}$, a new semiconducting transition-metal-dichalcogenide, epitaxially grown by direct selenization of Pt. Nano Lett. (2015) 15:4013-8. doi: 10.1021/acs.nanolett.5b00964

26. Yim C, Lee K, McEvoy N, O’Brien M, Riazimehr S, Berner NC, et al. High-performance hybrid electronic devices from layered $\mathrm{PtSe}_{2}$ films grown at low temperature. ACS Nano. (2016) 10:9550-8. doi: 10.1021/acsnano.6b 04898

27. Wagner S, Yim C, McEvoy N, Kataria S, Yokaribas V, Kuc A, et al. Highly sensitive electromechanical piezoresistive pressure sensors based on large-area layered $\mathrm{PtSe}_{2}$ films. Nano Lett. (2018) 18:3738-45. doi: 10.1021/acs.nanolett.8b00928

28. Yu X, Yu P, Wu D, Singh B, Zeng Q, Lin H, et al. Atomically thin noble metal dichalcogenide: a broadband mid-infrared semiconductor. Nat Commun. (2018) 9:545. doi: 10.1038/s41467-018-03935-0

29. Yim C, McEvoy N, Riazimehr S, Schneider DS, Gity F, Monaghan S, et al. Wide spectral photoresponse of layered platinum diselenide-based photodiodes. Nano Lett. (2018) 18:1794-800. doi: 10.1021/acs.nanolett.7b05000

30. Tao L, Huang X, He J, Lou Y, Zeng L, Li Y, et al. Vertically standing $\mathrm{PtSe}_{2}$ film: a saturable absorber for a passively mode-locked $\mathrm{Nd}$ : $\mathrm{LuVO}_{4}$ laser. Photon Res. (2018) 6:750-5. doi: 10.1364/PRJ.6.000750

31. Yuan J, Mu H, Li L, Chen Y, Yu W, Zhang K, et al. Few-layer platinum diselenide as a new saturable absorber for ultrafast fiber lasers. ACS Appl Mater Inter. (2018) 10:21534-40. doi: 10.1021/acsami.8b 03045

32. Zhang K, Feng M, Ren Y, Liu F, Chen X, Yang J, et al. Qswitched and mode-locked Er-doped fiber laser using $\mathrm{PtSe}_{2}$ as a saturable absorber. Photon Res. (2018) 6:893-9. doi: 10.1364/PRJ.6.0 00893

33. Geim AK, Novoselov KS. The rise of graphene. Nat Mater. (2007) 6:183 91. doi: $10.1038 /$ nmat 1849

34. Huang B, Du L, Yi Q, Yang L, Li J, Miao L, et al. Bulk-structured PtSe for femtosecond fiber laser mode-locking. Opt Express. (2019) 27:260411. doi: 10.1364/OE.27.002604

35. Zhang H, Tang DY, Zhao LM, Bao QL, Loh KP. Vector dissipative solitons in graphene mode locked fiber lasers. Opt Commun. (2010) b:3334-8. doi: 10.1016/j.optcom.2010. 04.064

36. Song YF, Shi XJ, Wu CF, Tang DY, Zhang $H$, et al. Recent progress of study on optical solitons in fiber 
lasers. Appl Phys Rev. (2019) 6:021313. doi: 10.1063/1.50 91811

37. Tang DY, Man R, Tam HY, Drummond P. Observation of bound states of solitons in a passively mode-locked fiber laser. Phys Rev A. (2001) 64:033814. doi: 10.1103/PhysRevA.64. 033814

38. Wang, Z, Xu Y, Dhanabalan SC, Ponraj J, Zhao C, Xu C, et al. Black phosphorus quantum dots as an efficient saturable absorber for bound soliton operation in an erbium doped fiber laser. IEEE Photon. J. (2016) 8, 5: 1-10. doi: 10.1109/JPHOT.2016.2598085
Conflict of Interest: The authors declare that the research was conducted in the absence of any commercial or financial relationships that could be construed as a potential conflict of interest.

Copyright $(02020 \mathrm{Wu}$ and Jiang. This is an open-access article distributed under the terms of the Creative Commons Attribution License (CC BY). The use, distribution or reproduction in other forums is permitted, provided the original author(s) and the copyright owner(s) are credited and that the original publication in this journal is cited, in accordance with accepted academic practice. No use, distribution or reproduction is permitted which does not comply with these terms. 